\title{
Underwater Low Contrast Color Images Enhancement based on Dynamic Thresholding and Stretching
}

\author{
Sukh Sehaj Singh \\ M.Tech Scholar \\ Department of Computer Engineering \\ Punjabi University, Patiala, India
}

\author{
Prof. Priyanka Jarial \\ Assistant Professor \\ Department of Computer Engineering \\ Punjabi University, Patiala, India
}

\begin{abstract}
In the modern era, the underwater images are captures to monitor the internal conditions of water. The underwater images capturing is difficult task because the water medium provides the different challenges like absorption, scattering, diffusion etc. To resolve these issues enhancement and color correction plays an important role. In this paper, the underwater images enhancement is done using exposure value, dynamic thresholding and stretching. The benefits of dynamic thresholding and stretching is that the naturalness of the image never loss and incontrollable manners the image stretched. Also, performance analysis of proposed technique is done with existing technique based on Qualitative and Quantitative analysis. The results reflect that the proposed technique have better enhancement and higher entropy as compared to existing technique.
\end{abstract}

Keywords: Underwater Image Enhancement, Dynamic Thresholding, Exposure Value, Entropy

\section{INTRODUCTION}

Light plays an important role in photography to generate the satisfactory quality images. The strong and weak light causes have a washed out appearance and poor visibility [1]. The underwater imaging is quite a challenging issue due to scattering, light absorption, reflection [2-3] as shown in fig. 1. These parameters are explain below

\section{- $\quad$ Scattering}

The scattering causes the change of the direction of the path. These causes generated not only due to water but also due to tiny particles in the water. There are two types of Scattering

- Forward Scattering

The Forward scattering causes the blurring of the images.

- Reverse Scattering

The backward scattering causes the contrast reduces [4].

- Color Absorption

The color wavelengths are absorbed in water deep water. The longest wavelength, with the lowest energy absorbed firstly.

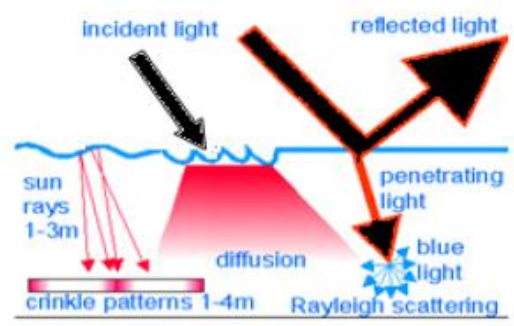

Figure 1 Reasons of distorted Underwater Images [3]
The quality or enhancement of underwater images is done using two ways.

- Image Restoration

The effect of degraded images can be minimized using filtering operations. The overall effectiveness of the process depends on the knowledge of degradation in the image. These techniques required many parameters such a attenuation and diffusion coefficients [5].

- Image Enhancement

The image enhancement techniques improve the visibility by enhancing the image component such as gamma value, color correction etc [5]. The image enhancement can be done by two ways

\section{- Histogram Stretching}

In histogram stretching techniques the histograms are stretched over maximum range for enhancement. The techniques are basically used for low contrast image enhancement and stretching technique remaps the histogram bins.

- Histogram Equalization

The histogram equalization technique equalizes the low contrast or high contrast image by uniform distribution of pixels using probability density function (PDF) and commutative distribution function (CDF).

The paper as follow the Section 2 defines the related work on underwater image enhancement, motivation and contribution. The Section 3 defines the proposed algorithm explanation. The section 4 defines the simulation results on MATLAB and Qualitative and Quantitative analysis is done. In last section V conclusion is done. 


\section{RELATED WORK}

In this section, a review on underwater image enhancement technique is done. Based on survey, defines motivation and our contribution in this paper.

Kuldeep Singh, et al. [6], proposed a recursive based histogram equalization technique for image enhancement. In their work, according to exposure value, thresholding sub-histogram created and histogram equalization is applied. The performance analysis of their work is done on the basis of qualitative and quantitative analysis (such as entropy). Also, comparative analysis is done with the existing techniques.

Ahmad Shahrizan Abdul Ghani and Nor Ashidi Mat Isa [7], worked on underwater image enhancement on the basis of histogram stretching. Their techniques have two steps contrast and color correction. In their first step, according to minimum and maximum histogram bins value mid-point found out. The left part of mid-point $5 \%$ increase stretching and right part of mid-point 5\%decrease stretching. In the second step color correction is done. The performance analysis is done on the basis of MSE, PSNR, Sobel count, MSSIM, EMEE, NIQE.

Ghani, et al. [8], proposed a hybrid technique in which contrast and color correction is done. The contrast stretching is done by multiplying the image by a gain factor and mid-point are found out based on maximum and minimum histogram bin. Further, color correction is done by transforming the RGB plane into HSV color space then $\mathrm{S}$ and $\mathrm{V}$ component enhanced.

Gao, et al. [9], discussed that they improving the underwater images by using the restoration and enhancement methods. In the initial stage, image restoration is done by estimating the atmospheric light, refining the transmittance image. After restoration process, the image enhancement is done using deduced histogram equalization method. Their experiment result shows that image quality improves effectively. $\mathrm{Xiu} \mathrm{Li}$, et al. [10], the underwater images are distorted due to scattering and color wavelength absorbed. They proposed a novel technique based on dark channel prior and luminance adjustment. Their technique resolves these two parameters issues and gives better visualization result.

\subsection{Motivation}

From the literature survey, the studies of Kuldeep Singh, et al. [6] shows that in their technique thresholding value is found out then all pixels are greater than threshold, all set to threshold value. Because of this operation no. of pixels losses of fine details or contained unwanted viewing arte-facts. In the paper, Ahmad Shahrizan Abdul Ghani and Nor Ashidi Mat Isa [7], based on the mid values stretching is done and if the image is under-contrast then mid values lie near 0 and their stretching technique not give enhancement.

\subsection{Contribution}

In our proposed technique, resolving these two issues. In our technique dynamic threshold is done which never losses fine details. Also, in our technique according to the exposure value stretching maximum limit is set which gives better enhancement as compared to existing techniques.

\section{Proposed Algorithm FOr IMAGe ENHANCEMENT}

In this section, the proposed algorithm and there description for image enhancement is given below.

1. Read the RGB image and histograms are plotted.
The underwater images are read for processing and their histograms are plotted for enhancement process.

2. Calculate exposure value by using equation 1 .

$$
\text { Exposure_value }=\frac{\sum_{k=0}^{L-1} h(k) * k}{L \sum_{k=0}^{L-1} h(k)}
$$

Where $\mathrm{h}(k)$ defines the histograms of the image.

$\mathrm{L}$ defines the total number of bins in the image (in gray scale images $\mathrm{L}$ value is 255).

The exposure value lies between 0 and 1 . If the value less than 0.5 the image have low contrast and if value higher than 0.5 the image have high contrast.

3. Dynamic thresholding of histogram bins

To maintain the naturalness in the image the thresholding process is done. In the existing algorithms proposed by Kuldeep, et al. [6], threshold value calculated by using equation 3.2 and the all values above than threshold are fixed to threshold value.

$T_{C}=\frac{1}{L} \sum_{k=0}^{L-1} h(k)$

$h_{c}(k)=h(k), h(k)<T_{C}$

$h_{c}(k)=T_{C}, h(k)>T_{C}$

Where $T_{C}$ is the threshold value calculated from histograms.

$\mathrm{h}(k)$ is the original histogram and $h_{c}(k)$ is the clipped histogram. In the proposed work, the dynamic threshold is done so, the histogram bins never loss a large amount of pixel values. The dynamic threshold is done by using equation 3.3.

$T_{C}=\frac{1}{L} \sum_{k=0}^{L-1} h(k)$

$h_{c}(k)=h(k), h(k)<T_{C}$

$h_{c}(k)=\frac{h(k)+T_{C}}{2}, h(k)>T_{C}$

4. Histogram Equalization

The clipped histogram equalization is done for enhancement.

5. Contrast stretching based on the exposure value.

The histogram equalized bins are stretched according to exposure value. The stretching also done variable by using equation 3.5

Stretching ${ }_{\text {Function }}=\frac{x(i, j)-\text { minimum }}{\text { maximum-minimum }} \mathrm{Xa}$

Where $i, j$ varies from 0 to size of image.

a: in place of 255 the a value is calculated using exposure value.

6. Qualitative and Quantitative Analysis of the proposed algorithm.

The performance analysis of proposed technique is done using qualitative and quantitative analysis. The parameters are calculated are Entropy, MSE, and PSNR.

7. Comparative analysis with existing results.

To show the performance of proposed technique is better than other a comparative analysis is done.

\section{RESULTS AND DISCUSSION}

The proposed technique is simulated on MATLAB 2013a software. The evaluated images databases taken from existing techniques qualitative analysis and 6 images results are shown here. The proposed technique Qualitative and Quantitative analysis is done and compared with existing technique is done.

The following Parameter is

Entropy: Entropy (Average information content) measures the proportions of the details of the frame and it is usually measured in units as bits.

$$
\mathrm{E}(\mathrm{p})=-\sum_{i=0}^{G-1} P(i) \log P(i)
$$


International Journal of Computer Applications Technology and Research

Volume 6-Issue 7, 344-348, 2017, ISSN:-2319-8656

Where $P(i)$ is probability density function of a given frame at a particular intensity level and $G$ is total number of grey levels in the frame. An frame having high entropy value is considered to be having high details and better quality.

Table 1 Qualitative and Quantitative Analysis and Comparison with existing techniques.

\begin{tabular}{|c|c|c|c|c|}
\hline Original Image & $\begin{array}{l}\text { Existing } \\
\text { Enhancement Image for Gray } \\
\text { Scale Images[6] }\end{array}$ & $\begin{array}{l}\text { Proposed } \\
\text { Enhancement Image }\end{array}$ & $\begin{array}{l}\text { Existing } \\
\text { Technique } \\
\text { Performance } \\
\text { Parameters }\end{array}$ & $\begin{array}{l}\text { Proposed } \\
\text { Technique } \\
\text { Performance } \\
\text { Parameters for } \\
\text { Color Images } \\
\end{array}$ \\
\hline Original Plane & R-ESIHE & Enhanced Image & $\begin{array}{l}\text { Original Entropy: } \\
5.05 \\
\text { Enhanced Image } \\
\text { Entropy }=5.04\end{array}$ & $\begin{array}{l}\text { Original } \\
\text { Entropy: } 5.25 \\
\text { Enhanced Image } \\
\text { Entropy=6.47 }\end{array}$ \\
\hline Original Plane & R-ESIHE & Enhanced Image & $\begin{array}{l}\text { Original Entropy: } \\
6.26 \\
\text { Enhanced Image } \\
\text { Entropy }=6.25\end{array}$ & $\begin{array}{l}\text { Original } \\
\text { Entropy: } 6.36 \\
\text { Enhanced Image } \\
\text { Entropy=6.97 }\end{array}$ \\
\hline Original Plane & R-ESIHE & Enhanced Image & $\begin{array}{l}\text { Original Entropy: } \\
5.87 \\
\text { Enhanced Image } \\
\text { Entropy }=5.85\end{array}$ & $\begin{array}{l}\text { Original } \\
\text { Entropy: } 6.05 \\
\text { Enhanced Image } \\
\text { Entropy }=6.27\end{array}$ \\
\hline & & & $\begin{array}{l}\text { Original Entropy: } \\
5.05 \\
\text { Enhanced Image } \\
\text { Entropy }=5.03\end{array}$ & $\begin{array}{l}\text { Original } \\
\text { Entropy: } 5.02 \\
\text { Enhanced Image } \\
\text { Entropy }=6.29\end{array}$ \\
\hline
\end{tabular}




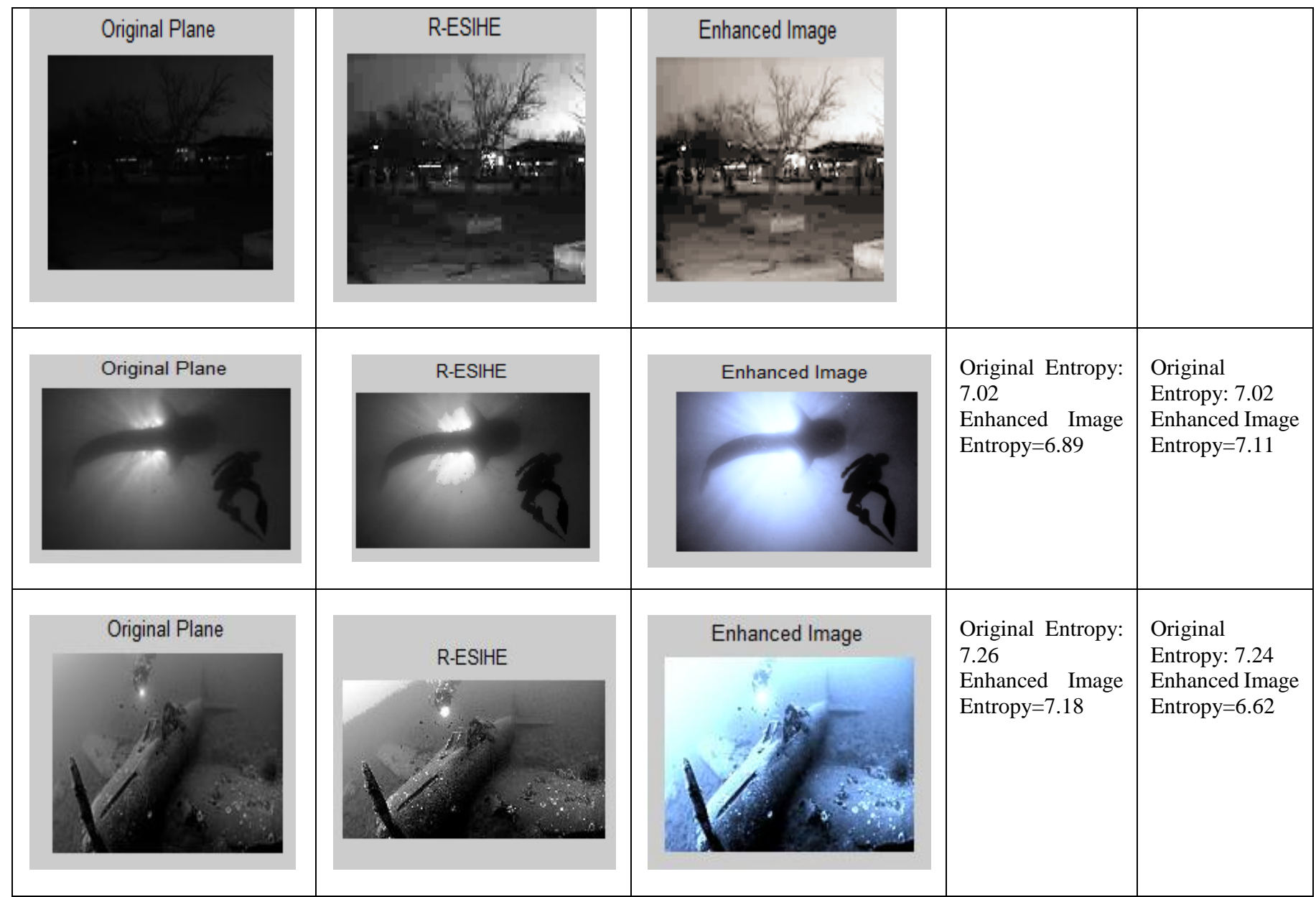

The table 1 shows that proposed technique have better enhancement as compared to existing algorithm. Also, quantitative analysis shows that the image performance parameter entropy is improved.

\section{CONCLUSION}

In this paper, the underwater image capturing issues are highlighted that the image quality degraded due to scattering, absorption. To resolve these issues a survey is done and based on survey a dynamic thresholding and stretching technique is proposed. The proposed algorithm is explained with their mathematical equations. The proposed technique qualitative and quantitative analysis is done and compare with existing technique. The results shows that proposed technique have better enhancement and high entropy as compared to existing techniques.

\section{REFERENCES}

[1] Ting, C.C. Wu, B.F. Chung, M.L. Chiu, C.C. Wu, Y.C. Visual, "Contrast Enhancement Algorithm Based on Histogram Equalization," Sensors, Vol. 15, pp. 16981-16999, , 2015.

[2] Hitam, M.S. Yussof, W.J. Awalludin, E.A. Bachok, Z. Mixture, "contrast limited adaptive histogram Equalization for Underwater Image Enhancement," International Conference on Computer Applications Technology, Vol. 1, pp.1-5, 2013.
[3] Vikram Dwivedi, Paresh Rawat, Nashrah Fatima, "Image Set Creation using Different Image Enhancement Technique for Underwater Image Segmentation," International Journal of Computer Applications, Vol. 150, No. 10, September 2016.

[4] Sowmyanshree M S, Sukrita K Bekal, Sneha, Priyanka, "A Survey on Various Underwater Image Enhancement Techniques," International Journal of Engineering Science Invention, Vol. 3, Issue 5, pp. 40-45, May 2014.

[5] R. C. Gonzalez and R. E. Woods, "Digital Image Processing", Reading. MA: Addison Wesley, 2004.

[6] Kuldeep Singh, Rajiv Kapoor, and Sanjeev Kr. Sinha, "Enhancement of low exposure images via recursive histogram equalization algorithms," Optik Journals, pp. 2619-2625, June 2015.

[7] Ahmed Shahrizan Abdul Ghani and Nor Ashidi Mat Isa, "Enhancement of low Quality Underwater Images through Integrated global and local contrast," Applied Soft Computing, pp.332-344, August 2015. 
International Journal of Computer Applications Technology and Research

Volume 6-Issue 7, 344-348, 2017, ISSN:-2319-8656

[8] Ahmad Shahrizan Abdul Ghani, Raja Siti Nur Adiimah Raja Aris, and Muhamad Luqman Muhd Zain, "Unsupervised Contrast Correction for underwater Image Quality Enhancement through Integrated Intensity Stretched-Rayleigh Histogram," Journal of Telecommunication, Electronic and Computer Engineering, Vol. 8, No. 3,pp. 1-7, 2016.
[9] Yakun Gao, Haibin Li, and Shuhuan Wen, "Restoration and enhancement of underwater images based on bright channel prior," Mathematical Problems in Engineering, pp. 1-15, September 2016.

[10] Xiu Li, Zhixiong Yang, and Min Shang, "Underwater image enhancement via dark channel prior and luminance adjustment," Shanghai Ocean, April 2016. 